\title{
reconnaissance par diagraphies des fractures recoupées par un forage
}

\author{
survey by various logging techniques of natural fractures \\ intersecting a borehole
}

\author{
F.H. CORNET \\ Laboratoire de Sismologie, \\ Institut de Physique du Globe de Paris*
}

Rev. Franç. Géotech. n` 45, p.p. $5 \cdot 19$ (octobre 1988)

\section{Résumé}

Dans le cadre d'une expérimentation in-situ centrée sur l'étude des possibilités d'exploiter la chaleur des roches profondes peu perméables, différentes techniques ont été mises en œuvre pour reconnaître les fractures naturelles recoupées par des forages de $800 \mathrm{~m}$ de profondeur implantés dans un massif granitique.

La diagraphie électrique Mosnier a permis de préciser avec efficacité le pendage et l'azimut des fractures. Les résultats des diagraphies d'atténuation des ondes mécaniques hautes fréquences (Darcilog, atténuation des ondes S et atténuation des ondes de Stoneley) susceptibles de fournir une caractérisation des propriétés hydrauliques des fractures ont été confrontés à ceux de débitmétries et thermométries obtenues lorsque les forages étaient soit en condition d'injection soit en condition de production. Aucune de ces diagraphies, à elle seule, n'a permis de mettre en évidence systématiquement toutes les fractures significatives du point de vue des écoulements forcés dans le massif.

Ces résultats permettent de conclure que sur la centaine de fractures reconnues pour chacun des forages, moins d'une dizaine est d'importance pour les écoulements forcés. Ainsi il est apparu qu'il ne suffit pas de mesurer la conductivité hydraulique des fractures au niveau des forages mais qu'il est également nécessaire de reconnaître leur interconnection à l'intérieur du massif.

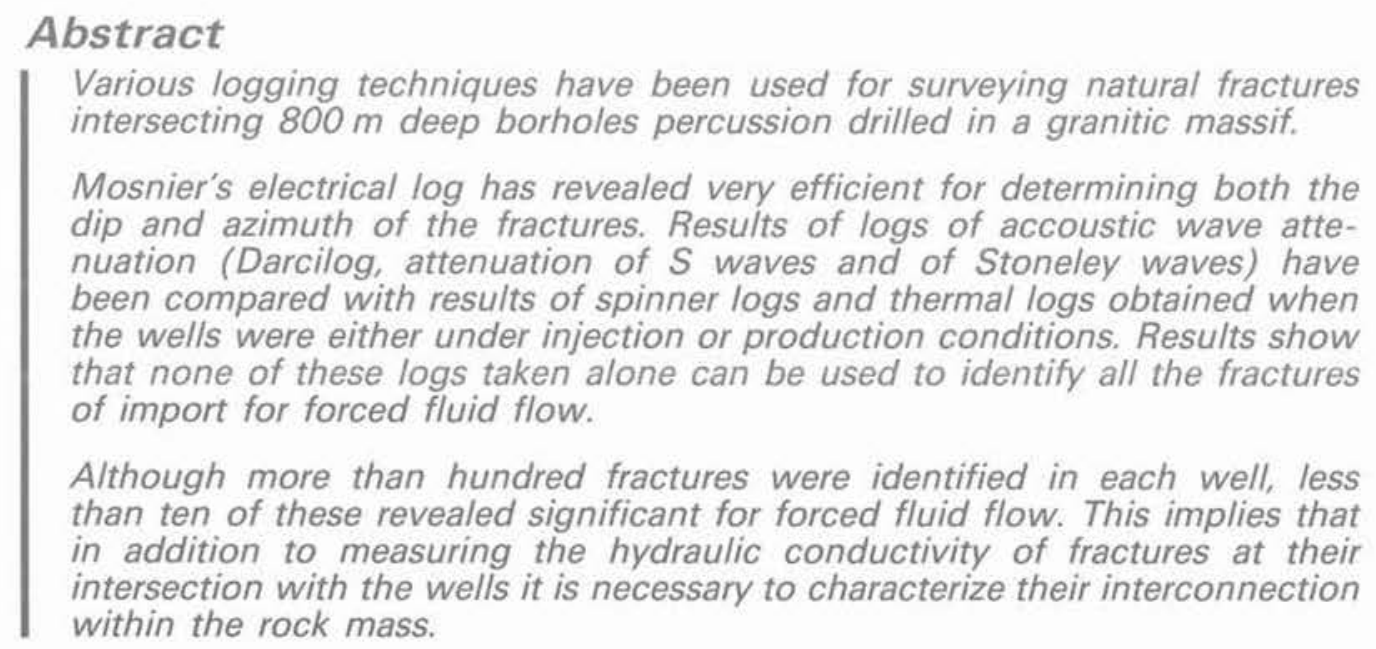

- 4 place Jussieu, Tour 14, 75252 Paris Cedex 05, Tél 43.36.25.25 


\section{INTRODUCTION}

Un programme de recherche, centré sur l'étude in-situ des possibilités de développer un échangeur thermique artificiel dans un massif cristallin, se poursuit depuis 1984 sur le site granitique du Mayet de Montagne, à $25 \mathrm{~km}$ au sud-est de Vichy.

L'objectif était de faire circuler des débits d'eau de l'ordre de $60 \mathrm{~m}^{3} / \mathrm{h}$ entre deux forages (INAG III-8 et INAG III-9) distants l'un de l'autre de $100 \mathrm{~m}$ et profonds d'environ $800 \mathrm{~m}$. Dans ce cadre, un effort a été porté sur l'identification par méthodes géophysiques des fractures naturelles qui recoupent les forages et sur la caractérisation de leurs propriétés hydrauliques.

Cet article présente les résultats de diverses diagraphies (thermiques, électriques, acoustiques et géochimiques) réalisées dans le forage INAG III-8 ainsi que les débitmétries et thermométries réalisées lorsque ce forage était placé en condition d'injection ou de production.

Une attention particulière a été portée sur les possibilités d'évaluer la conductivité hydraulique des fractures à partir de diagraphies acoustiques. Cette discussion se place exclusivement sur la base observationnelle fournie par la confrontation des résultats déduits des diagraphies acoustiques (d'une part Darcilog, d'autre part diagraphie d'atténuation des ondes $\mathrm{S}$ et diagraphies d'atténuation des ondes de Stoneley obtenues à partir des résultats de la sonde EVA de Elf-Aquitaine) avec les résultats des débitmétries et thermométries.

Le forage INAG III-8 profond de $782 \mathrm{~m}$ a été réalisé au marteau fond de trou. Son diamètre est de $250 \mathrm{~mm}$ jusqu'à $170 \mathrm{~m}$ de profondeur, puis de $165 \mathrm{~mm}$ jusqu'à $550 \mathrm{~m}$ et enfin de $159 \mathrm{~mm}$ jusquà $782 \mathrm{~m}$. Il était tubé de la surface jusqu'à $170 \mathrm{~m}$ lors des premières diagraphies mais, du fait de déficiences dans le tubage, ce dernier a été retiré par la suite.

L'inclinaison de l'axe du forage vis-à-vis de la verticale est inférieure à $3^{\circ} 5$ jusqu'à $680 \mathrm{~m}$, profondeur à partir de laquelle cette inclinaison augmente progressivement pour atteindre $8^{\circ}$ à $750 \mathrm{~m}$. De $750 \mathrm{~m}$ à $782.7 \mathrm{~m}$ (longueur totale forée) l'inclinaison est constante et égale à $8^{\circ}$ dans la direction $\mathrm{N} 148^{\circ} \mathrm{E}$.

Une diagraphie par diamétreur à 4 bras indique que le forage est bien calibré et circulaire sur toute sa hauteur sauf entre les cotes $221,5 \mathrm{~m}$ et $228 \mathrm{~m}$ où sa forme devient irrégulière. Le diamètre moyen atteint $240 \mathrm{~mm}$ entre $221,5 \mathrm{~m}$ et $224 \mathrm{~m}$.

\section{ETUDE DES «CUTTINGS »}

La remontée des cuttings s'est faite à l'air seul jusqu'à $14 \mathrm{~m}$ et, à partir de ce niveau, par une émulsion d'eau et d'air comprimé, avec addition d'un produit moussant aux plus grandes profondeurs. Les cuttings ont été prélevés à des fins d'analyses (COUTURIÉ et al., 1984) chaque fois que le forage avait progressé de $1 \mathrm{~m}$. Pour vérifier la représentativité de ces échantillons, des analyses chimiques comparatives ont également été réalisées par ces auteurs sur des carottes du sondage
INAG III- 1 et sur des cuttings de INAG III-3 pour des cotes identiques $(28 \mathrm{~m}, 84 \mathrm{~m}$ et $110 \mathrm{~m})$; ces deux sondages sont à moins d'une quinzaine de mètres de INAG III-8. La comparaison des résultats ne montre pas de variations significatives, mis à part une diminution de 10 à $20 \%$ de Feo et Mgo sur certains échantillons de cuttings (attribuable à un entraînement par l'eau des petites lamelles de biotite).

Le forage traverse sur toute sa hauteur le granite du Mayet de Montagne (décrit par PEYREL, 1981). Il s'agit d'un monzogranite porphyroïde contenant des méga. cristaux de feldspath potassique de teinte rose dont la taille varie de 3 à $5 \mathrm{~cm}$. La biotite (10\% en vol.) est associée à la hornblende verte (1 à $2 \%$ ) contenant parfois des reliques de clinopyroxène. On note deux facies de granite, le premier correspond à la roche saine et le second à la roche rubéfiée. Le faciès rubéfié, qui correspond à une hydrothermalisation du granite, est dominant (65\% du forage); il a été traversé sans interruption de $469 \mathrm{~m}$ jusqu'au fond du forage.

Des variations de composition minéralogique indiquant un changement lithologique (concentration feldspathique; enclaves microgrenues sombres correspondant à une abondance de minéraux ferromagnésiens) s'observent à différentes profondeurs.

La fracturation naturelle s'est manifestée durant le forage par des chutes d'outil ou des vitesses d'avancement très importantes accompagnées parfois de venues d'eau. Dans les cuttings elle se traduit par la présence de fragments de brêche quartzo-phylliteuse verdâtre ou de cataclasite noire. A certaines fractures sont associés des sulfures (chalcopyrite ou pyrite) ainsi que de la calcite ou du quartz hydrothermal.

\section{ANALYSE GÉOCHIMIQUE DES EAUX DU FORAGE}

L'analyse géochimique des eaux du forage a été réalisée par BIDAUX (1987). Des échantillons de $300 \mathrm{~cm}^{3}$ ont été prélevés tous les $20 \mathrm{~m}$, de 180 à $780 \mathrm{~m}$. Les mesures du $\mathrm{PH}$ et de l'alcalinité $\left(\mathrm{HCO}_{3}{ }^{-}\right)$ont été effectuées sur le terrain alors que les dosages concernant $\mathrm{SiO}_{2}, \mathrm{Cl}^{-}, \mathrm{NO}_{3}^{-}, \mathrm{SO}_{4}^{--}, \mathrm{Ca}^{++}, \mathrm{Na}^{+}, \mathrm{Li}$ et $\mathrm{Mn}$ ont été réalisés au baboratoire.

Il apparaît que l'eau est de facies dominant bicarbonaté calcique légèrement acide (PH variant de 6.52 à 6.93). Les variations de composition $(\mathrm{mg} / 1)$ au long du forage sont en général faibles et irrégulières (voir fig. 1).

On relève cependant un niveau réducteur à $480 \mathrm{~m}$, lié très probablement à une circulation d'eau, et à la présence de sulfures, et des teneurs anormalement faibles en $\mathrm{Na}^{+}$et $\mathrm{K}^{+}$à $580 \mathrm{~m}$ qui seraient dues à la présence de granite altéré.

L'analyse comparée des différentes courbes de variation de composition avec la profondeur a amené BIDAUX à conclure à l'existence de circulations d'eau, assurée à $480 \mathrm{~m}$, très probables à $540 \mathrm{~m}$ et entre 660 et $680 \mathrm{~m}$. 


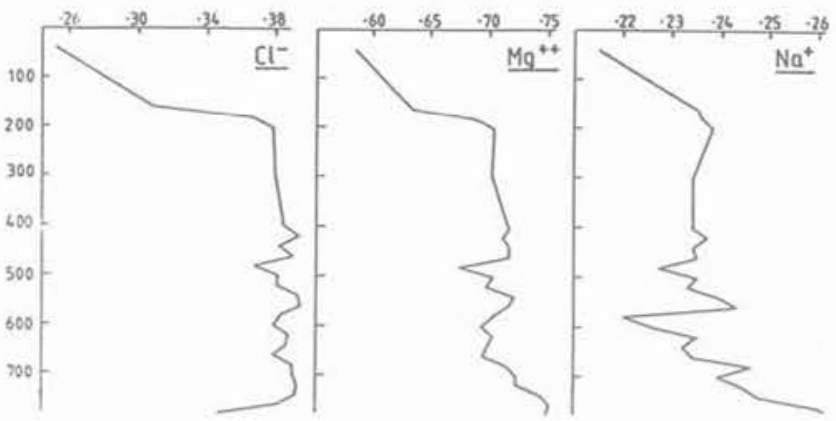

Fig. 1. - Variations avec la profondeur (exprimée en métres) de la teneur en $\mathrm{Cl}, \mathrm{Mg}^{++}$et $\mathrm{Na}^{+}$(exprimées en milligrammes par litres) de l'eau du forage (d'après Bidaux, 1987)

Fig. 1. - Variation with depth of various ions contents in the water filling the well. Depth is expressed in meters. ion contents are expressed in milligrams per liter (after Bidaux, 1987)

En outre des possibilités de circulation naturelle sont proposées à $200 \mathrm{~m}$, entre $260 \mathrm{~m}$ et $280 \mathrm{~m}$, à $360 \mathrm{~m}$ et à $380 \mathrm{~m}$ et peut être aussi entre $609 \mathrm{~m}$ et $610 \mathrm{~m}$ ainsi qu'à $720 \mathrm{~m}$.

\section{DIAGRAPHIES THERMIQUES DU FORAGE AU REPOS}

Une diagraphie thermique a été réalisée 45 jours après la fin du forage, période pendant laquelle le puits est resté au repos (JOLIVET, 1984). Le tubage était encore en place dans les $170 \mathrm{~m}$ superficiels. Ce dernier, du fait des nombreuses discontinuités qu'il présentait, a endommagé la liaision sonde thermique-câble au cours de la descente de la sonde de sorte que le gradient géothermique n'a pu être mesuré au-delà de $500 \mathrm{~m}$.

Au cours de cette diagraphie la température a été mesurée avec une thermistance unique ayant une constante de temps inférieure à la seconde, une sensibilité supérieure au millième de degré et une fidélité de lordre du centième de degré.

Différents traitements numériques peuvent être appliqués à ces mesures de température (JOLIVET, 1984). Nous montrons sur la figure 2.a les variations avec la profondeur du gradient moyen calculé sur une base de 5 mètres. On remarque qu'il est à peu près égal à $30^{\circ} / \mathrm{km}$ entre $250 \mathrm{~m}$ et $375 \mathrm{~m}$ mais qu'il a tendance à augmenter avec la profondeur. Les faits les plus marquants sont cependant les anomalies positives ou négatives, observées à $192 \mathrm{~m}$, de 220 à $240 \mathrm{~m}$, à $364 \mathrm{~m}$, à $410 \mathrm{~m}$ et surtout l'anomalie de $473 \mathrm{~m}$ jusqu'à $490 \mathrm{~m}$. Cette anomalie correspond à une augmentation notoire du gradient qui atteint $55^{\circ} / \mathrm{km}$; la température du forage à $490 \mathrm{~m}$ de profondeur était de $24^{\circ}$.

Cette diagraphie a été reprise en 1985 après que le tubage ait été retiré de la partie supérieure du forage, neuf mois après des essais d'injection réalisés, le pre-

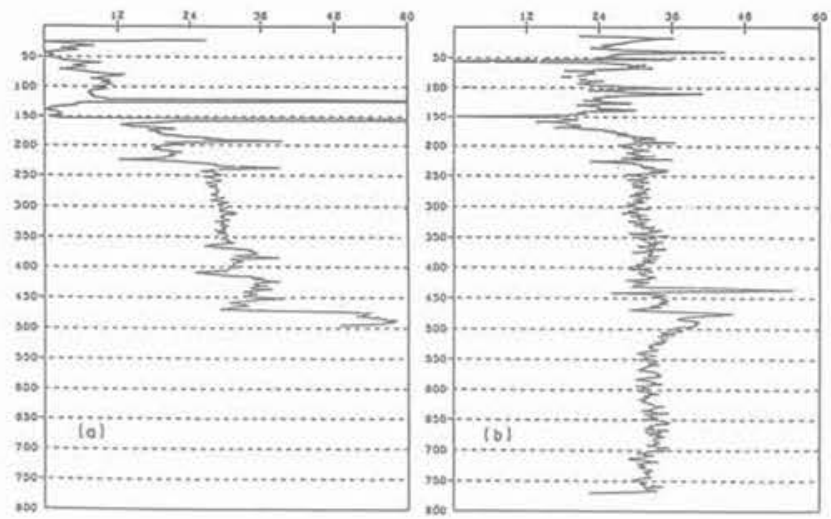

Fig. 2. - Diagraphies thermiques (d'après Jolivet, 1986): variations avec la profondeur du gradient géothermique moyen (en degrés Celsius par kilomètre) calculé sur une longueur de $5 \mathrm{~m}$ avec une mesure tous les $25 \mathrm{~cm}$, a) diagraphie obtenue un mois après la fin du forage; b) diagraphie obtenue neuf mois après qu'une stimulation hydraulique ait eu lieu à $441 \mathrm{~m}$.

Fig. 2. - Thermal log of the well at rest (after Jolivet. 1986), i.e. variation with depth of the mean geothermal gradient (expressed in degree celsius per kilometer) computed for a $5 \mathrm{~m}$ base with temperature measurements every $25 \mathrm{~cm}$.a) $\mathrm{log}$ of the well as observed one month after drilling. b) log of the well as observed nine months after a hydraulic stimulation was performed at $441 \mathrm{~m}$.

mier à $317 \mathrm{~m}$ avec des débits très faibles $\left(3 \mathrm{~m}^{3} / \mathrm{h}\right)$, le second à $441 \mathrm{~m}$ à des débits élevés $\left(1.2 \mathrm{~m}^{3} / \mathrm{min}\right)$. Les variations avec la profondeur du gradient moyen calculé sur une base de $5 \mathrm{~m}$ sont présentés sur la figure 2.b.

On remarque que la partie supérieure du forage (jusqu'à $200 \mathrm{~m}$ ) est très perturbée mais que le gradient moyen devient beaucoup plus régulier en-dessous si ce n'est pour deux accidents majeurs à $441 \mathrm{~m}$ et $473 \mathrm{~m}$. Les fortes anomalies positives observées en 1984 à $190 \mathrm{~m}$ et $225 \mathrm{~m}$ ont nettement diminué. Il apparaît donc que les essais d'injection à $441 \mathrm{~m}$ ont perturbé de façon significative le gradient thermique de ce forage et par conséquent que les anomalies observées sont liées à des circulations d'eau et non à des variations de conductivité thermique.

L'anomalie positive détectée à $473 \mathrm{~m}$ est sensible jusqu'à $550 \mathrm{~m}$ de profondeur ce qui indique qu'il existait à cette cote, avant le forage, un flux d'eau ascendant relativement important. Ce flux est pratiquement entièrement capté par les fractures développées à $441 \mathrm{~m}$ ce qui entraîne une diminution des anomalies observées à $225 \mathrm{~m}$ et $190 \mathrm{~m}$.

L'absence d'anomalie en dessous de $550 \mathrm{~m}$ suggère que s'il existe des écoulements naturels d'eau dans le réseau de fractures recoupé en-dessous de cette cote, les vitesses d'écoulement y sont suffisamment faibles pour que l'eau soit en équilibre thermique avec l'en. caissant.

Ces résultats confirment donc ceux des diagraphies géochimiques pour ce qui est d'un écoulement d'eau à $480 \mathrm{~m}$ mais permettent de conclure que s'il y a écoulement à $540 \mathrm{~m}$ et aux environs de $670 \mathrm{~m}$, comme 
le suggèrent les diagraphies géochimiques, les vitesses y sont très faibles.

Le gradient moyen mesuré entre $175 \mathrm{~m}$ et $772 \mathrm{~m}$ s'établit à $32.5^{\circ} / \mathrm{km}$ valeur qui est pratiquement la même que celle mesurée dans le forage INAG III-4 entre $180 \mathrm{~m}$ et $250 \mathrm{~m}$ de profondeur.

\section{DIAGRAPHIES ÉLECTRIQUES}

Un certain nombre de diagraphies électriques ont été réalisées, d'une part selon les méthodes classiques de lindustrie pétrolière (Latérolog et pendagemétrie de SCHLUMBERGER), d'autre part, avec la sonde MOS. NIER spécialement développée pour ce programme.

Le latérolog mesure les variations de résistivité électrique pour des «tubes » de roche coaxiaux au forage et dont l'épaisseur est une fonction de la distance entre les électrodes. La pendagemétrie correspond à des mesures de résistivité électrique dans quatre azimuts différents au moyen de patins, disposés aux extrémités de deux diamètres perpendiculaires entre eux, frottant contre la paroi. Par corrélation entre les résultats obtenus pour chacun des patins, il est possible de calculer le pendage de la structure conductrice recoupée par le forage en supposant que celle-ci soit plane. Cette diagraphie est couplée à celle de la détermination de la géométrie du forage (azimut et pendage de l'axe du forage, variations de diamètre dans deux directions perpendiculaires entre elles).

Ces diagraphies sont classiques et ne seront pas discutée plus avant. Notons simplement que les résultats obtenus avec ces deux techniques sont tout à fait comparables à ceux de la diagraphie électrique MOS. NIER quant à la détermination des profondeurs des fractures recoupées par le forage mais que la diagraphie MOSNIER s'est révélée beaucoup plus précise pour la détermination des pendages et azimuts des fractures.

Le principe de la diagraphie électrique MOSNIER (MOSNIER, 1981) utilisée pour cartographier les fractures consiste à mesurer la différence de conductivité électrique entre la matrice rocheuse (résistante) et le matériau qui remplit la fracture (plus conducteur du fait de sa teneur élevée en eau). Une carte de l'impédance électrique de la paroi du forage est obtenue en mesurant l'impédance à la fois en fonction de l'azimut et de la profondeur. Cette diagraphie est comparable à la pendagemétrie de SCHLUMBERGER mais la sonde MOSNIER permet une «cartographie» de la totalité de la paroi du forage contrairement à la pendagemétrie qui ne fournit ces informations que pour les secteurs balayés par les 4 patins.

Une sonde de 26 électrodes ( 2 de garde et 24 de mesures) a été construite (voir fig. 3). Le courant est injecté dans le terrain par la sonde, l'électrode de recueil étant l'armature métallique externe du câble de diagraphie. Pour obliger les lignes de courant issues des électrodes de mesures à pénétrer dans le terrain, au lieu de se refermer immédiatement sur le câble, une certaine longueur de ce dernier, juste au-dessus de la sonde, est isolée par une gaine. Les deux électrodes

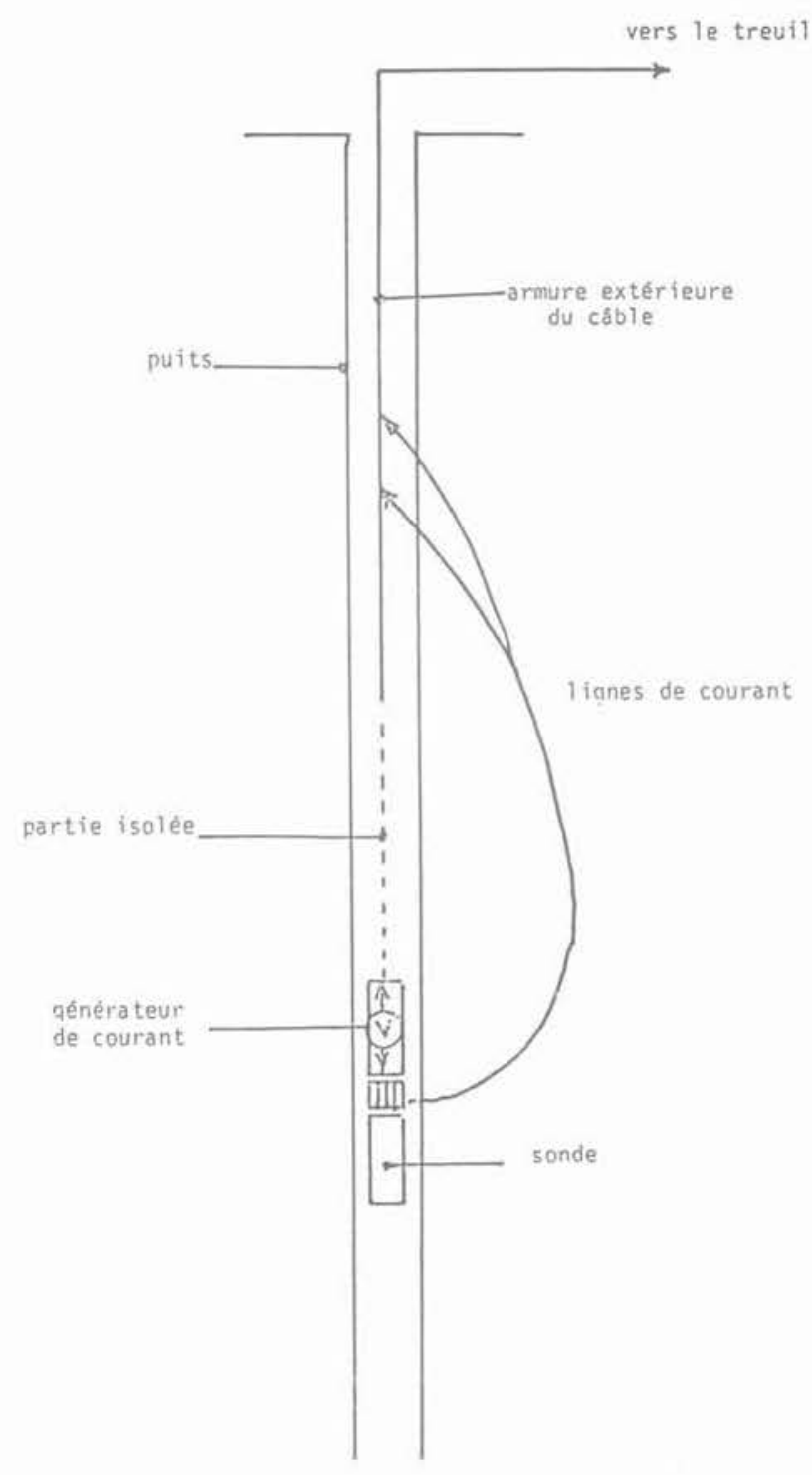

Fig. 3. - Schéma de fonctionnement de la sonde électrique Mosnier.

Fig. 3. - Schematic diagram of Mosnier's electrical sonde.

de garde, disposées de part et d'autre des 24 électrodes de mesures, permettent d'assurer que les lignes de courant au niveau des électrodes de mesures sont parfaitement normales à la paroi du forage. Les électrodes de mesures permettent de déterminer l'intensité du courant qui pénètre dans la formation dans tous les azimuts (une électrode de mesure tous les $15^{\circ}$ ), l'orientation de la sonde étant repérée par rapport au Nord magnétique.

Un exemple de résultat obtenu entre les cotes $472 \mathrm{~m}$ et $470 \mathrm{~m}$ est présenté sur la figure 4 . On a représenté les amplitudes de l'intensité du courant mesuré pour les 24 électrodes sur des diagrammes polaires.

On remarque plus particulièrement l'apparition d'un conducteur dans la direction n $70^{\circ} \mathrm{E}$ à $471.9 \mathrm{~m}$. Ce conducteur se dédouble ensuite pour des cotes décroissantes puis réapparaît solitaire à $470.7 \mathrm{~m}$ dans la direc- 

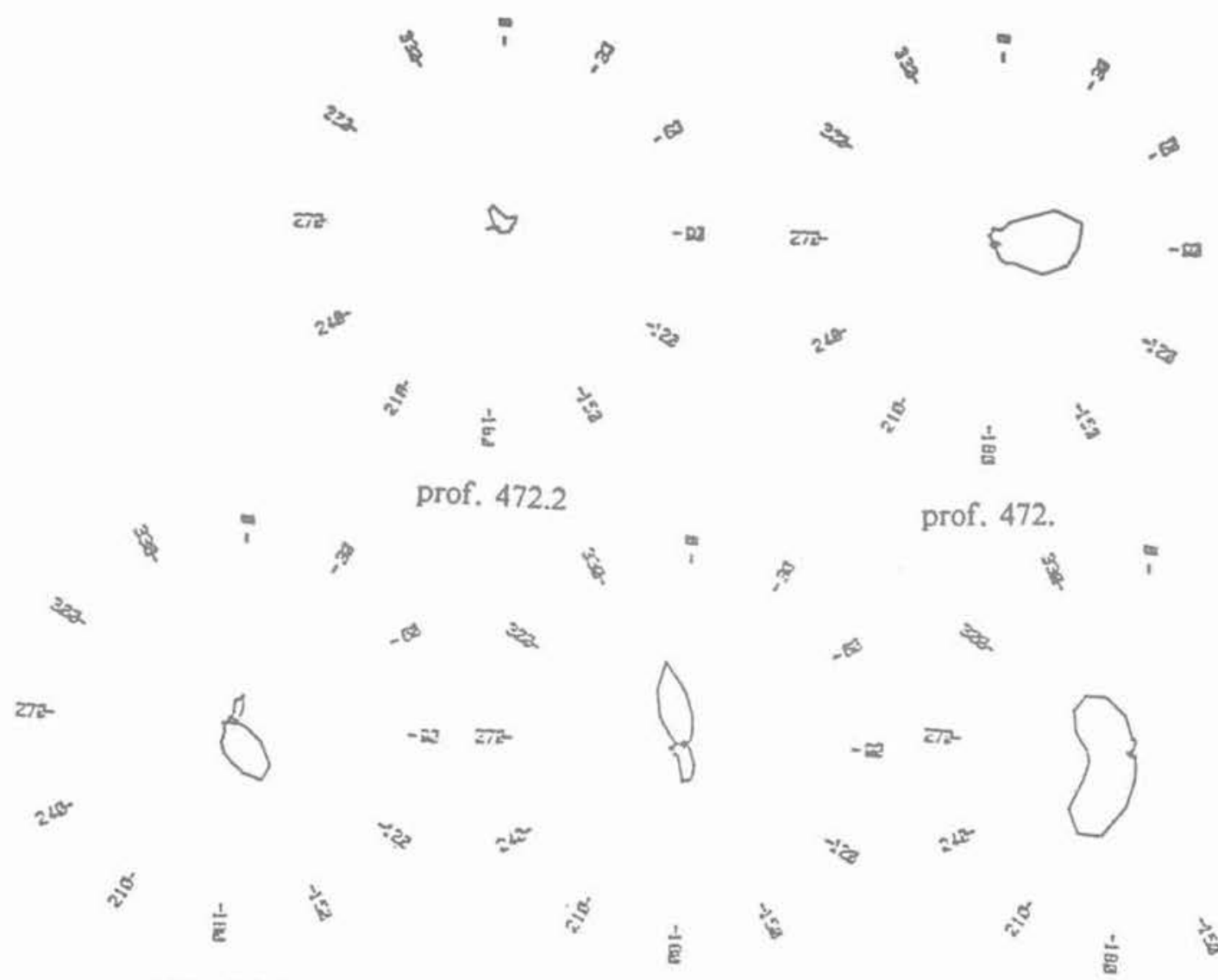

prof. 472.

prof. 471.8

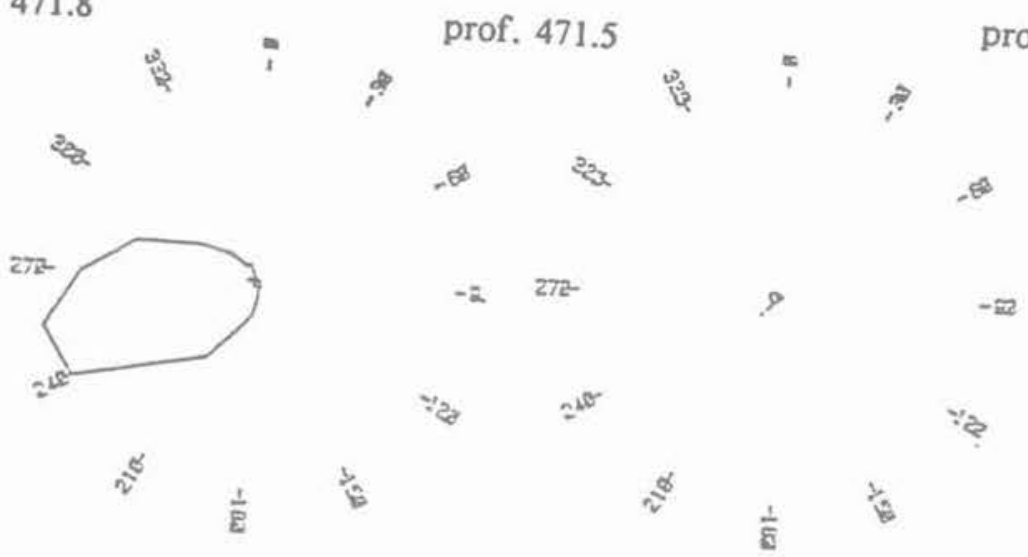

prof. 470.7

prof. 470.5

Fig. 4. - Exemple de diagramme polaire des variations azimutales normées de l'intensité électrique mesurées entre 472 et $470 \mathrm{~m}$.

tion $\mathrm{N} 250^{\circ} \mathrm{E}$ et disparait pour les cotes supérieures. Ces résultats permettent d'identifier une fracture dans la direction $\mathrm{N} 160^{\circ} \mathrm{E} \pm 7^{\circ}$ et de pendage $85^{\circ}$ (fracture sub-verticale). Elle correspond à la cote où était apparue l'anomalie thermique significative décrite ci-dessus.

Un deuxième type de présentation des résultats consiste à associer à chaque groupe de 24 valeurs de l'intensité $\mathrm{i}(\mathrm{z}), 24$ carrés dont la noirceur dépend de lintensité émise par l'électrode correspondante. Une nouvelle
Fig. 4. - Polar diagram of azimuthal variations of electrical intensity (normalized with respect to the maximum value measured in the well) as observed with Mosnier's electrical tool between $472 \mathrm{~m}$ and $470 \mathrm{~m}$.

ligne est tracée pour la cote $z+\Delta z$, où $\Delta z$ est l'incrément de profondeur entre deux scrutations azimutales et ainsi de suite. On obtient ainsi une cartographie électrique de la paroi du forage. Un exemple de résultat est présenté sur la figure 5 où apparaît nettement la fracture à $471 \mathrm{~m}$. Cette cartographie du forage est obtenue en temps réel sur le terrain.

Cette diagraphie a été réalisée sur la hauteur totale du forage et il serait fastidieux d'énumérer ici l'ensemble 


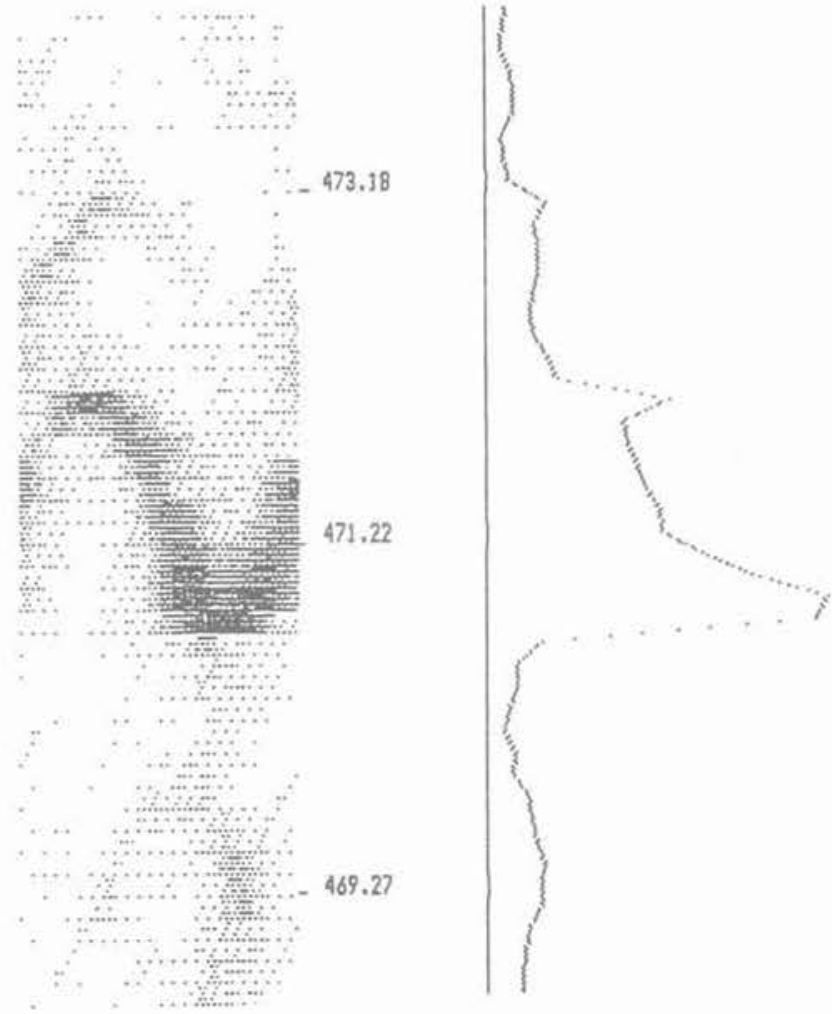

Fig. 5. - Image électrique du forage entre 472 et $470 \mathrm{~m}$. La courbe de droite représente le logarithme de lintensité totale du courant injecté.

Fig. 5. - Electrical image of the well between 472 and $470 \mathrm{~m}$. The right hand curve represents the logarithm of the total injected electrical intensity

des fractures mises en évidence (plus d'une centaine). Seule une approche statistique de ces résultats peut permettre d'obtenir une représentation de la fracturation naturelle ainsi observée. Cet aspect ne sera pas développé ici.

Soulignons que seules les fractures conductrices d'électricité sont mises en évidence avec cette méthode, et que toutes celles qui sont recimentées ne peuvent pas être cartographiées de la sorte. Afin d'identifier les niveaux les plus significatifs du point de vue de ces mesures électriques, nous avons relevé toutes les cotes où la résistance mesurée avec le laterolog LLD de SCHLUMBERGER était inférieure à 100 ohms (voir tableau 2).

\section{DIAGRAPHIES ACOUSTIQUES}

Les diagraphies acoustiques consistent à mesurer la manière dont évolue avec la profondeur la propagation des ondes mécaniques haute fréquence (généralement dans le domaine de la vingtaine ou de la trentaine de kilohertz pour les ondes P) au voisinage du forage.

Le principe est le suivant (voir par exemple ROSENBAUM, 1974; PAILLET and WHITE, 1982): une source émet une onde de compression dans le fluide qui remplit le forage. Cette onde induit dans la roche des ondes de volume $\mathrm{P}$ et $\mathrm{S}$, des ondes de surface pseudo Rayleigh et pseudo Stoneley) qui se propagent à, ou au voisinage de l'interface fluide-solide et des ondes de compression dans le fluide. Les ondes de volume et les ondes de surface induisent à leur tour des ondes de compression dans le fluide, de sorte qu'elles peuvent être détectées par des capteurs de pression placés dans le fluide (hydrophones).

A condition de disposer de moyens d'acquisition de données suffisamment rapides pour être compatibles avec le domaine de fréquence des signaux observés, il est possible d'enregistrer la totalité du train d'ondes. De tels enregistrements permettent de déterminer les temps d'arrivée des différents types d'onde ainsi que l'atténuation qui leur est associée, si l'on dispose de deux ou plusieurs récepteurs.

De nombreux travaux théoriques ont été centrés sur l'étude de la propagation des ondes haute fréquence dans et au voisinage d'un forage rempli de fluide, creusés dans des matériaux poreux et/ou fracturés, saturés par un ou plusieurs fluides (voir par exemple ROSENBAUM 1974; CHENG and TOKZÖZ, 1981 ; PAILLET and WHITE, 1982 ; WHITE 1983 ; MATHIEU and TOKSÖZ, 1984; BEYDOUN et al., 1985; RA. SALOFOSAON, 1987 ; HARDIN et al., 1987).

Il n'est pas de notre propos de mener ici une étude détaillée, théorique, de ce problème. Nous nous contenterons de préciser que toutes ces études font ressortir le fait que l'atténuation des différentes ondes observées, en accord avec la théorie de BIOT (1956), dépend notamment du mouvement du, ou des fluides(s) dans le réseau de pores et donc de la perméabilité du milieu.

LEBRETON et al. (1978) et CONCHE et al. (1986) semblent convaincus que l'onde $\mathrm{P}$ est la plus appropriée pour étudier la perméabilité du milieu. PAILLET and WHITE (1982), RASALOFOSAON (1987), parmi d'autres, ont montré que l'atténuation des ondes $\mathrm{P}$ était beaucoup moins sensible à la perméabilité du milieu que celle des ondes $\mathrm{S}$ ou que celle des ondes de STONELEY. Ces auteurs préconisent d'étudier l'atténuation des ondes de STONELEY, toujours bien marquées, pour extraire une information quantitative concernant la conductivité hydraulique des fractures plutôt que l'atténuation des ondes $\mathrm{S}$ du fait, pour ces dernières, des arrivées quasi-simultanées des ondes pseudo RAYLEIGH pour certains milieux.

MATHIEU et TOKSÖZ (1984) ont étudié les relations qui existent entre l'atténuation des ondes de STONE. LEY et l'épaisseur hydraulique d'une fracture recoupant le forage perpendiculairement à son axe. HARDIN et al. (1987) présentent, pour un massif granitique, une comparaison entre des mesures directes de perméabilité effectuées en isolant une portion de forage entre obturateurs et les valeurs de conductivité hydraulique déduites des logs d'atténuation des ondes de STONELEY. Les résultats qu'ils présentent suggèrent qu'il est effectivement possible d'apprécier la conductivité hy draulique des fractures à partir des diagraphies d'atténuation des ondes de STONELEY.

\subsection{Darcilog}

Dans le cadre des études menées au Mayet de Montagne, nous avons tout d'abord fait réaliser une 
diagraphie sonique par la société SCHLUMBERGER, selon les normes prescrites par F. LEBRETON, pour obtenir un Darcilog du forage III-8 (distance émetteur. premier récepteur égale à $90 \mathrm{~cm}$ (3 pieds) et distance premier récepteur - deuxième récepteur égale à $60 \mathrm{~cm}$ ( 2 pieds), acquisition numérique à un pas d'échantillo. nage de 4 microseconde avec une dynamique de l'ordre de $40 \mathrm{db}$ ). Le Darcilog correspond aux variations avec la profondeur de lindice I défini (LEBRETON et al., 1978) par le rapport $A_{2}+A_{3} / A_{1}$, où $A$ est l'amplitude de la ième arche du signal associé à Ponde $\mathrm{P}$ avec $\mathrm{I}$ $=1,2,3$. Cet indice est aussi parfois défini par un rapport de pentes (voir CONCHE, 1986 et fig. 6).

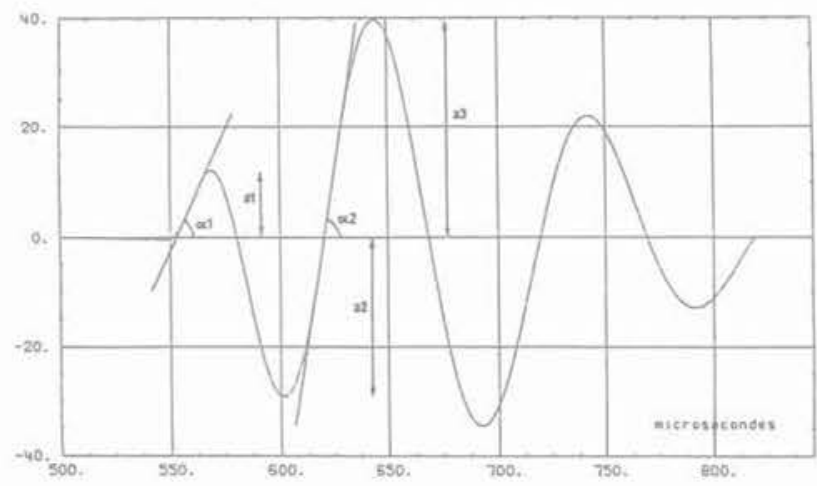

Fig. 6. - Définition de l'indice I utilisé pour le Darcilog (d'après Conche, 1986). Cet indice est défini soit par un rapport d'amplitudes $\left(a_{2}+a_{3}\right) / a_{1}$, soit par un rapport de pentes $\left(\operatorname{tg} \alpha_{2} / \operatorname{tg} \alpha_{1}\right)$.

Fig. 6. - Definition of the the $I_{c}$ index used in Darcilog (after Conche, 1986). Ic is defined either as the amplitude ratio $\left(a_{2}+a_{3}\right) / a_{1}$ or as the slope ratio $\operatorname{tg} \alpha_{2} / \operatorname{tg} \alpha_{1}$.

L'indice I dépend de latténuation du signal et donc aussi de la perméabilité du milieu. D'après DUCOMTE et CONRAD (1984), la relation suivante serait vérifiée:

$$
I_{c}=\alpha \log (k / \mu)+\beta
$$

ou : $\alpha$ et $\beta$ sont des constantes caractéristiques de la sonde acoustique ;

$\mu$ est la viscosité du fluide dans la roche;

$\mathrm{k}_{\mathrm{v}}$ est la composante verticale de la perméabilité du milieu (si le forage est vertical).

On remarquera ainsi que d'après la relation (1) la perméabilité verticale d'une fracture horizontale dans un massif granitique étant nulle, l'indice $I_{c}$ devrait être insensible aux fractures horizontales.

Les résultats obtenus, présentés sur la figure 7, sont discutés plus précisément dans la section 8 lors de leur confrontation avec les résultats des autres diagraphies et plus particulièrement ceux des débitmètres et thermométries.

\subsection{Sonde EVA}

Indépendamment de ce travail, la Société Elf Aquitaine (Département sismique de puits) a réalisé pour son compte un certain nombre de diagraphies acoustiques dans le forage III-8 à l'aide de la sonde EVA (MATHIEU et ARDITTY, 1986). La sonde EVA (Evaluation des Vitesses et Atténuations) est équipée de cinq émetteurs (dans le domaine $3-25 \mathrm{Khz}$ ) distants l'un de l'autre de $0,25 \mathrm{~m}$ et de douze récepteurs distants l'un de l'autre de $1 \mathrm{~m}$, permettant de travailler avec des distances émetteur-récepteur variant de $1 \mathrm{~m}$ à $13 \mathrm{~m}$.

Le signal complet, reçu par tous les récepteurs, était enregistré numériquement avec un pas d'échantillonnage de 5 micro secondes et 12 bits par échantillon (soit une dynamique de $72 \mathrm{db}$ ). Cette acquisition permet de connaître le temps d'arrivée et l'amplitude des différents types d'ondes sur les différents récepteurs.

A partir des amplitudes des ondes $S$ et de celles des ondes de STONELEY, MATHIEU et ARDITTY ont calculé des diagraphies de rapports d'amplitude pour deux recepteurs distant l'un de lautre de $1 \mathrm{~m}$ (voir

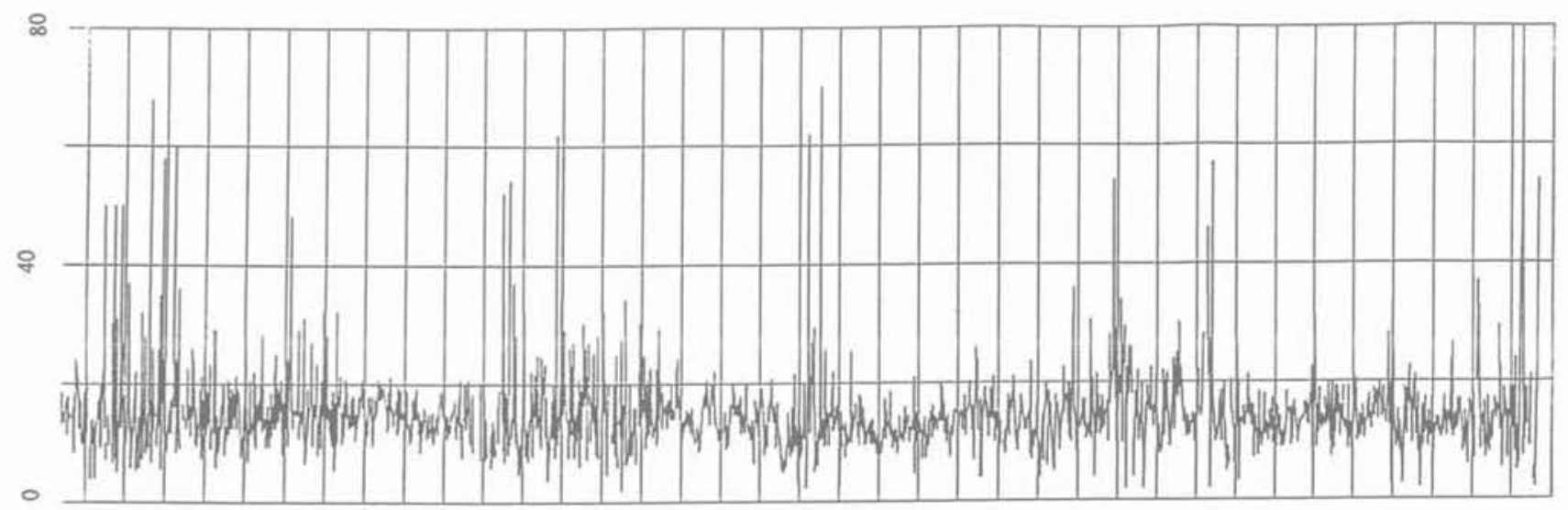

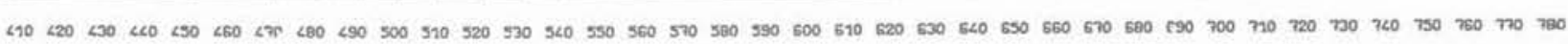

Fig. 7. - Darcilog du forage INAG III-8 avant toute stimulation hydraulique.
Fig. 7. - Darcilog of well INAG III-8 before any hydraulic stimulation. 
$+1.1$

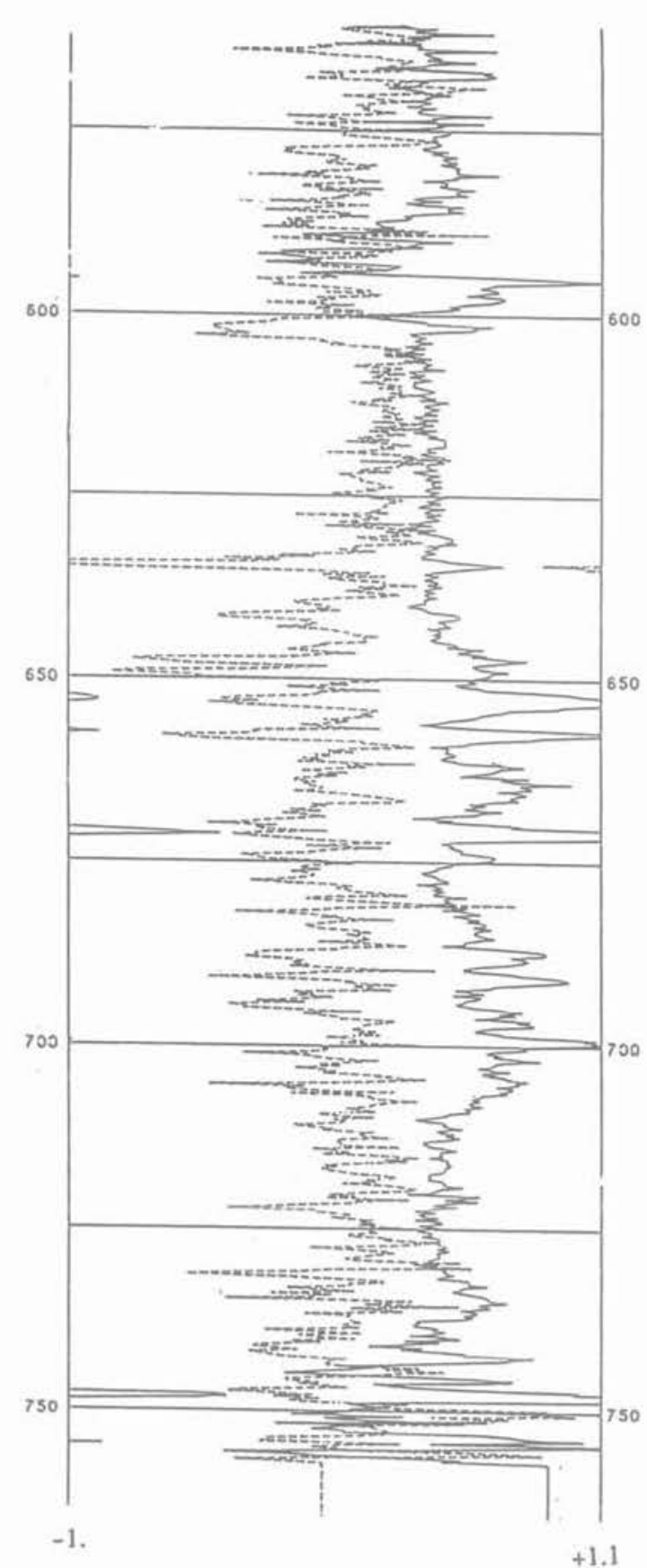

-1 . 600

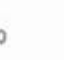

$+1.1$

Fig. 8. - Diagraphies d'atténuation des ondes $S$ (courbe en pointillés à gauche, variations entre +1.1 (à gauche) et -1 ) et des ondes de Stoneley (courbe en trait plein à droite, variation entre -1 (à gauche) et +1.1 ). L'atténuation est ici simplement représentée par les rapports d'amplitude du même signal vu sur deux récepteurs distants l'un de l'autre de $1 \mathrm{~m}$, d'après MATHIEU et ARDITTY, 1986.

Fig. 8. - Log of $S$ wave and Stoneley wave attenuation (after Mathieu and Arditty, 1980). S wave attenuation (dotted curve on the left) varies between +1.1 (on the left) to -1 : Stoneley wave attenuation (curve on the right) varies between -1.0 (on the left) and +1.1 . Attenuation (adimentional) is computed as the ratio between amplitudes of the signal as observed on two sensors distant from each other by one meter. fig. 8) ce qui permet d'obtenir des diagraphies caractéristiques de l'atténuation dans le terrain pour ces types d'onde.

HARDIN et al. (1987) et MATHIEU et ARDITTY proposent des solutions pour extraire de ces diagraphies d'atténuation des valeurs de perméabilité. Toutefois nous préférons nous en tenir aux résultats bruts et comparer les cotes des zones déclarées très perméables à partir de ces diagraphies à celles déduites des résultats des débitmétries et thermométries réalisées lorsque le forage était en condition d'injection ou de production.

Afin de mettre en évidence les zones induisant les plus fortes atténuations pour les ondes $S$ et pour les ondes de STONELEY, nous avons défini un seuil en fonction du niveau de bruit et donnons dans le tableau 2 les cotes pour lesquelles l'atténuation (en fait le rapport d'amplitude) mesurée atteint des valeurs au moins trois fois supérieures à ce seuil. On remarque que les seules cotes qui aient été mises en évidence communément par ces deux diagraphies sont : $170 \mathrm{~m}, 192 \mathrm{~m}, 221$ $223 \mathrm{~m}, 657.5 \mathrm{~m}$, et $706.5 \mathrm{~m}$.

Ces résultats correspondent à une diagraphie enregistrée en 1984 c'est-à-dire avant les essais d'injection d'eau réalisés à $317 \mathrm{~m}$ et $441 \mathrm{~m}$. Par comparaison avec des diagraphies du même type réalisées après ces essais d'injection (voir fig. 9) on remarque que la stimulation hydraulique à $441 \mathrm{~m}$ a entraîné une très forte atténuation des ondes S mais n'a quasiment pas modifié l'atténuation des ondes de STONELEY. Avant fracturation hydraulique, une fissure préexistante sub-horizontale peu marquée avait été identifiée sur la diagraphie élec. trique MOSNIER à $441 \mathrm{~m}$. Après fracturation hydraulique, une empreinte orientée du forage a révélé trois fractures sub-horizontales et une fracture verticale. Nous avons ainsi une démonstration expérimentale simple que toutes les fractures ne sont pas détectées par les diagraphies d'atténuation des ondes de STONELEY mais que, peut-être par combinaison avec les diagraphies d'atténuation des autres ondes, il est possible d'identifier tous les niveaux hydrauliquement conducteurs. Nous reviendrons sur cette proposition lors de la confrontation des différents résultats.

\section{DÉBITMÉTRIES ET THERMOMÉTRIES}

Afin de préciser les zones les plus conductrices hydrauliquement, des débitmétries et thermométries ont été effectuées durant des essais d'injection et de production d'eau.

\subsection{Débitmétrie en condition d'injection}

Un train de tiges se terminant à $251 \mathrm{~m}$ de profondeur par un obturateur gonflable placé entre ce train de tiges et la paroi du forage, a permis de réaliser en dessous de cette cote une injection d'eau. Le débit d'injection a été égal à $21 \mathrm{~m}^{3} / \mathrm{h}$ durant les six premières minutes de l'essai puis a été maintenu égal à $30.5 \mathrm{~m}^{3} / \mathrm{h}$. La pression en tête de puits a crû progressivement de 3.4 $\mathrm{MPa}$ au début de la débitmétrie à $4.1 \mathrm{MPa}$ à la fin de cette dernière. 


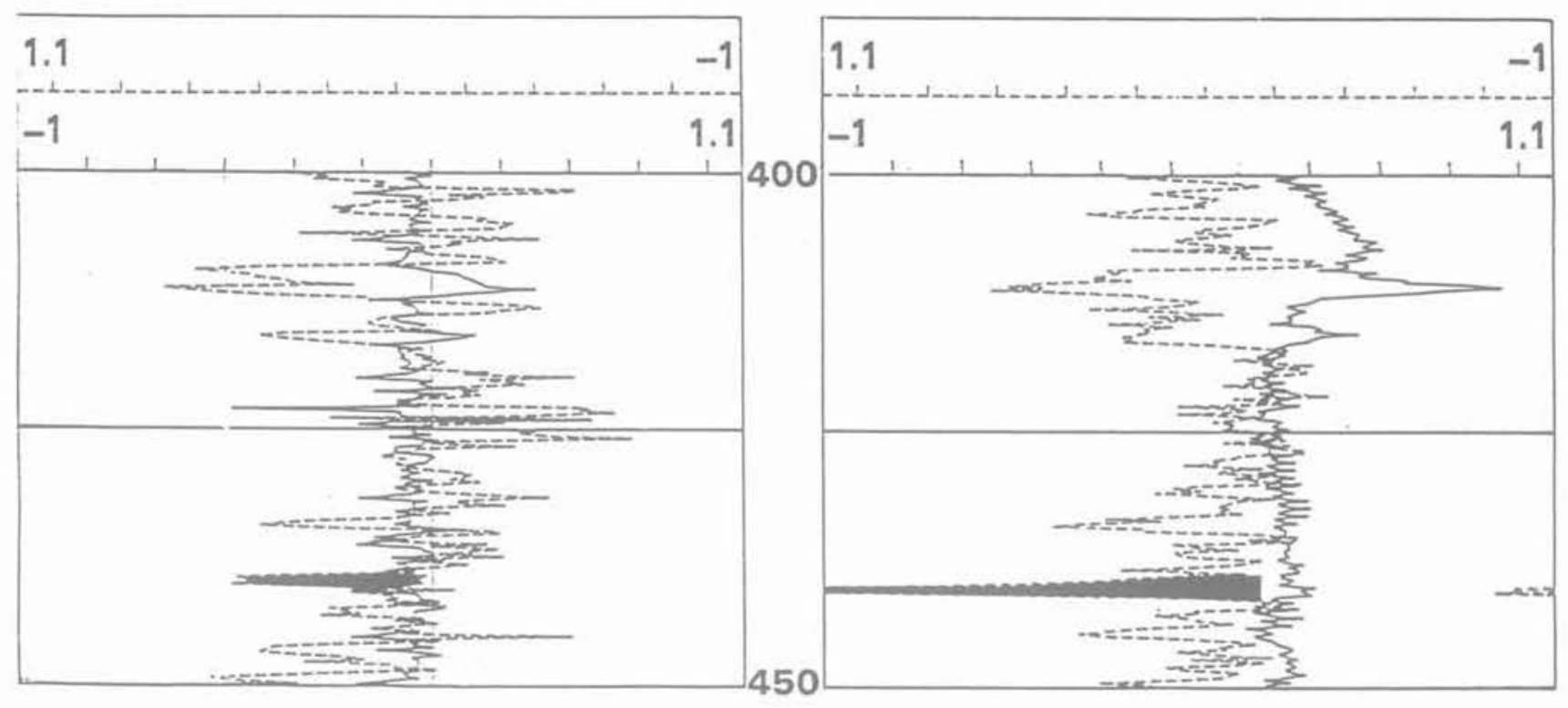

a)

Fig. 9. - Diagraphies d'atténuation des ondes $S$ et des ondes de Stoneley (d'après Mathieu et Arditty, 1986). a) diagraphies réalisées avant la stimulation hydraulique à $441 \mathrm{~m}$; b) diagraphies réalisées après la stimulation hydraulique à $441 \mathrm{~m}$

La mesure des variations avec la profondeur du débit d'écoulement dans le forage a été réalisée en mesurant la vitesse de rotation d'un micro-moulinet pour une vitesse moyenne de remontée de l'appareil de $20.8 \mathrm{~m} /$ min.

Des calibrations ont montré qu'en labsence de tout écoulement dans le forage, la vitesse de rotation du micro-moulinet est linéairement dépendante de la vitesse de remontée de l'outil. En outre, lorsque le micromoulinet se déplace dans un flux descendant constant, la droite d'étalonnage vitesse de rotation - vitesse de remontée subit une simple translation ce qui montre bien que laccroissement de vitesse de rotation du micro-moulinet est proportionnelle au débit injecté.

Les variations de vitesse de rotation avec la profondeur sont présentées sur la figure 10.a. On y remarque plus particulièrement les cotes $267,441 \mathrm{~m}, 472 \mathrm{~m}, 633 \mathrm{~m}$ et $638 \mathrm{~m}$ pour lesquelles des variations de débit bien marquées sont observées ainsi qu'une décroissance régulière du débit de 638 à $700 \mathrm{~m}$.

En fait l'anomalie observée à $638 \mathrm{~m}$ ne correspond pas à un écoulement dans une fracture mais à la variation du débit d'injection destinée à corriger les dérives de

l'unité de pompages. Comme cela a été mentionné cidessus le débit d'injection est passé de $21 \mathrm{~m}^{3} / \mathrm{h}$ à $30.4 \mathrm{~m}^{3} / \mathrm{h}$.

Il serait souhaitable d'extraire de ces résultats des informations quantitatives concernant l'impédance hydraulique des fractures. Nous évaluerons cette grandeur par le rapport entre, d'une part la différence qui existe
Fig. 9. - Log of $S$ wave and Stoneley wave attenuation observed. a) before and b) after a hydraulic stimulation at $441 \mathrm{~m}$ (after Mathieu and Arditty. 1986).

entre la pression dans le forage à cette cote et la pression interstitielle à l'infini dans le massif à la même cote et, d'autre part, le débit injecté dans la fracture.

Si l'on en juge par la profondeur des niveaux d'eau dans les différents forages réalisés sur ce site, la pression interstitielle dans le milieu avant toute injection est égale à la pression hydrostatique (le niveau d'eau se trouve à une profondeur variant de 3 à $6 \mathrm{~m}$ de la surface du sol selon les forages). La différence entre la pression dans le forage à la cote de la fracture et la pression dans le massif à cette profondeur est donc égale à la pression d'injection en tête du forage.

Cependant, la débitmétrie a été effectuée en condition non stationnaire. Elle a débuté 2 à 3 heures après le démarrage du pompage alors que la stationnarité des conditions d'écoulement n'aurait été atteinte que 3 à 4 jours après le début du pompage ainsi que cela a été observé lors d'essais ultérieurs. Aussi ces mesures d'impédance ne sont elles qu'indicatives qualitativement des fractures les plus permissives.

De plus, il a été observé que la réponse du forage au changement de débit d'injection intervenu dans la débitmétrie n'est pas linéaire. En effet la vitesse de rotation du micro-moulinet lors des essais de calibration entre 350 et $400 \mathrm{~m}$ de profondeur, réalisés avant la diagraphie avec un débit d'injection inférieur à $30 \mathrm{~m}^{3} / \mathrm{h}$, atteignait 7.8 tours $/ \mathrm{sec}$ mais elle n'était que de 7.2 tours/sec dans le même intervalle lorsque la débitmétrie a été réalisée à un débit d'injection de $30.4 \mathrm{~m}^{3} / \mathrm{h}$. Ce résultat indique que la différence de débit d'injection a été entièrement accomodée par l'écoulement à $268 \mathrm{~m}$. Cette observation trouve sa justification dans le 


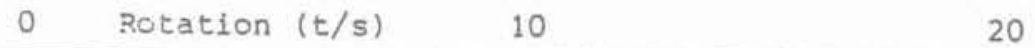

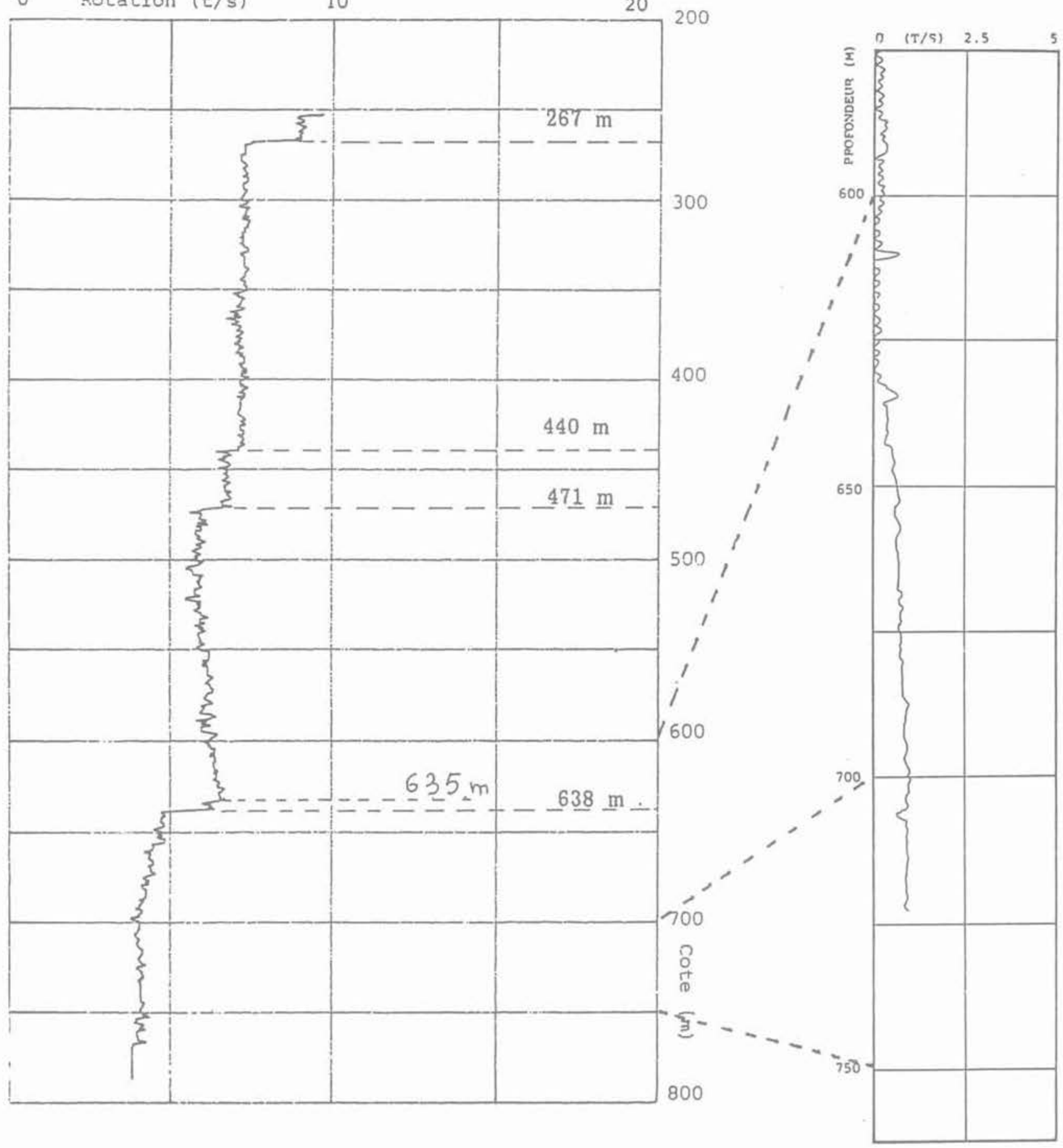

Fig. 10. - Débitmétrie du forage placé a) en condition d'injection b) en condition de production. L'axe horizontal représente les variations de vitesse de rotation du micro-moulinet (exprimées en tours par seconde).

fait qu'à cette profondeur la pression dans le forage lors de la débitmétrie $(6.8 \mathrm{Mpa})$ était à peu près égale au poids des terrains $(7.0 \mathrm{MPa})$ et supérieure à la contrainte principale minimum $(5.6 \mathrm{MPa})$ alors qu'elle n'était que de $5.3 \mathrm{MPa}$ lors des essais de calibration. Ceci laisse supposer que l'augmentation de pression de $1.5 \mathrm{MPa}$ a permis d'ouvrir une ou plusieurs fractures à $268 \mathrm{~m}$ de profondeur ce qui a induit une diminution
Fig. 10. - Spinnerlog (flow meter) of the well under a) injection conditions and b) production conditions. Horizontal axis represents the variations of rotation velocity of a micro propeller (expressed in rotation per second).

d'impédance hydraulique de la paroie du forage à cette cote. Cette proposition est confirmée par l'observation, sur la diagraphie électrique MOSNIER réalisée avant la débitmétrie, d'une fracture sub-horizontale à $268 \mathrm{~m}$ de profondeur.

On remarque que l'ouverture de cette fracture à sa dimension finale n'est pas instantanée étant donné 
l'augmentation de débit observée à $638 \mathrm{~m}$ simultanément à l'accroissement du débit d'injection en tête de puits. Au cours des cing minutes qui ont suivi cette augmentation du débit d'injection (c'est-à-dire lorsque le micro-moulinet s'est déplacé de la cote $633 \mathrm{~m}$ à la cote $525 \mathrm{~m}$ ) la fracture à $268 \mathrm{~m}$ a vu son impédance hydraulique chuter du fait de son ouverture progressive ce qui a induit une diminution de débit injecté dans le forage en dessous de cette cote, d'où la diminution apparente de débit d'écoulement dans le forage lorsque le micro-moulinet s'est déplacé de $635 \mathrm{~m}$ à $525 \mathrm{~m}$.

Ces résultats montrent qu'il est extrêmement malaisé de déduire de cette débitmétrie des valeurs significatives pour l'impédance hydraulique des fractures. On peut cependant, pour chacune des fractures où intervient une perte d'eau, évaluer le changement de vitesse de rotation du micro-moulinet. Les résultats sont fournis dans le tableau 1. Ainsi que cela a été montré lors des
Du fait qu'il y a eu un changement de débit d'injection en cours de mesure, la somme des débits injectés dans les différentes fractures n'est pas égale au débit injecté en tête de puits à la fin ou au début des essais.

\subsection{Débitmétrie et thermométrie en condition de production}

Afin de préciser les zones hydrauliquement connectées au réseau de fractures naturelles, nous avons injecté de l'eau à $60 \mathrm{~m}^{3} / \mathrm{h}$ dans le forage III-9 situé à $100 \mathrm{~m}$ de III.8 et réalisé, dans III-8, une thermométrie et une débitmétrie lorsque ce forage a produit un débit détectable avec le micro-moulinet.

TABLEAU 1. - Evaluation des impédances hydrauliques des fractures mises en évidence par la débitmétrie pour le forage INAG III-8

TABLEAU 1. - Evaluation of hydraulic impedance of fractures from the spinnerlay run during injection

\begin{tabular}{|c|c|c|c|}
\hline $\begin{array}{c}\text { Profondeur } \\
(\mathrm{en} \mathrm{m})\end{array}$ & $\begin{array}{c}\text { Variation de vitesses } \\
\text { de rotation tour/seconde }\end{array}$ & $\begin{array}{c}\text { Débit injecté } \\
\mathrm{m}^{3} / \mathrm{h}\end{array}$ & $\begin{array}{c}\text { Impédance hydraulique } \\
\mathrm{MPa} / \mathrm{m}^{3} / \mathrm{h}\end{array}$ \\
\hline 268 & 1,8 & 8,6 & 0,5 \\
440 & 0,5 & 2,4 & 1,7 \\
473 & 1,0 & 4,8 & 0,9 \\
635 & 0,5 & 2,3 & 1,6 \\
656 & 0,35 & 1,6 & 3,1 \\
673 & 0,25 & 1,1 & 3,8 \\
687 & 0,20 & 0,9 & 0,5 \\
\hline
\end{tabular}

calibrations, la différence de vitesse de rotation du micro-moulinet, observée entre la situation où il se déplace à une vitesse $\mathrm{V}$ dans le forage lorsqu'aucun écoulement n'y intervient et la situation où un débit d'injection $\mathrm{Q}$ existe dans le forage pour la même vitesse de remontée $V$ de l'outil, est proportionnelle à ce débit $\mathrm{Q}$. Ce facteur de proportionnalité $\eta$ a été évalué à partir des mesures de vitesse de rotation effectuées au-dessus de $265 \mathrm{~m}$ lors de la diagraphie, c'est-à-dire avant toute perte dans des fractures mais pour un même rapport entre le diamètre de la sonde et celui du forage $\left(\eta=4,8 \mathrm{~m}^{3} / \mathrm{h}\right.$ pour une vitesse de rotation de 1 tour par seconde). En dessous de $550 \mathrm{~m}$, le diamètre du forage est égal à $159 \mathrm{~mm}$ et le facteur de proportionnalité devient $4.5 \mathrm{~m}^{3} / \mathrm{h}$ pour une vitesse de rotation de 1 tour par seconde.

Pour les fractures observées au-dessus de $765 \mathrm{~m}$ de profondeur, le débit injecté dans ces fractures est obtenu simplement en multipliant la différence entre les vitesses de rotation observées de part et d'autre de la fracture par le facteur de proportionnalité $\eta$. L'impédance hydraulique est alors évaluée en divisant la pression d'injection en tête de puits par ce débit. Le débit injecté globalement dans les fractures qui recoupent le forage en dessous de $765 \mathrm{~m}$ (cote du début de la débitmétrie) a été évalué en soustrayant à la vitesse de rotation observée la valeur correspondant à la vitesse de remontée de l'outil lorsque le forage est au repos.
Les mesures ont été effectuées entre $730 \mathrm{~m}$ et $510 \mathrm{~m}$ pour la débitmétrie lavec une vitesse de remontée de $11 \mathrm{~m} / \mathrm{min}$.) et entre $773 \mathrm{~m}$ et $450 \mathrm{~m}$ pour la thermométrie. Il est à souligner qu'un tubage était présent dans le forage jusqu'à $525 \mathrm{~m}$ de profondeur, cote où un obturateur gonflable était supposé obstruer l'annulaire mais qui, du fait de son non gonflement, laissait en réalité l'eau s'écouler à l'extérieur du tubage.

La débitmétrie (voir fig. 10.b) permet de déduire que l'écoulement entre $635 \mathrm{~m}$ et $525 \mathrm{~m}$ est constant si ce n'est peut être une très faible venue d'eau vers $580 \mathrm{~m}$ (variation de vitesse de rotation du micro-moulinet légèrement inférieure à $0,2 \mathrm{t} / \mathrm{sec}$.). Des venues d'eau sont notées à $635 \mathrm{~m}$ (la plus importante), $647.5 \mathrm{~m}$ et $687.5 \mathrm{~m}$. Ces trois niveaux produisent ensemble un débit induisant une variation de vitesse de rotation d'environ $0,9 \mathrm{t} / \mathrm{sec}$. Aucune calibration du micro-moulinet n'a malheureusement été effectuée lors de ces mesures de sorte qu'il n'est pas possible d'évaluer les débits correspondants.

La thermométrie (voir fig. 11) met bien en évidence les niveaux où interviennent des venues d'eau, la diagra. phie de gradient thermique fournissant un piqué des cotes plus précis. On relève ainsi entre $510 \mathrm{~m}$ et $770 \mathrm{~m}$ les cotes $635 \mathrm{~m}, 648 \mathrm{~m}, 653 \mathrm{~m}, 671 \mathrm{~m}, 687.5 \mathrm{~m}$, $691 \mathrm{~m}, 699 \mathrm{~m}$ et $700 \mathrm{~m}$. Aucune venue d'eau n'est identifiée vers $580 \mathrm{~m}$ ce qui suggère que la faible 
$-0.3$

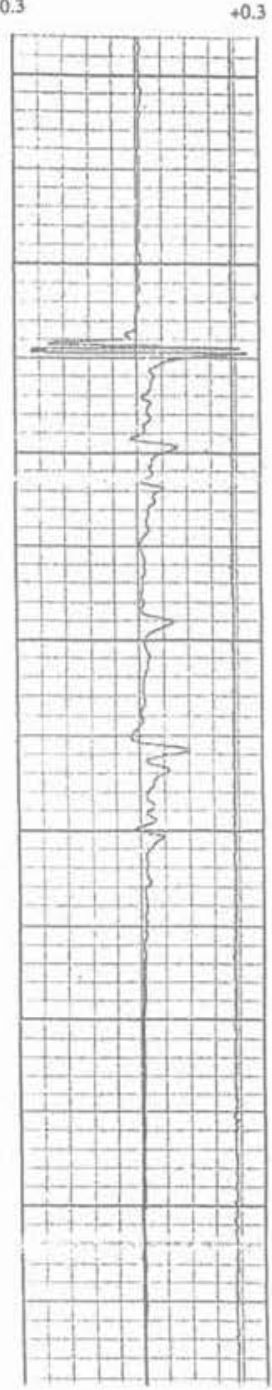

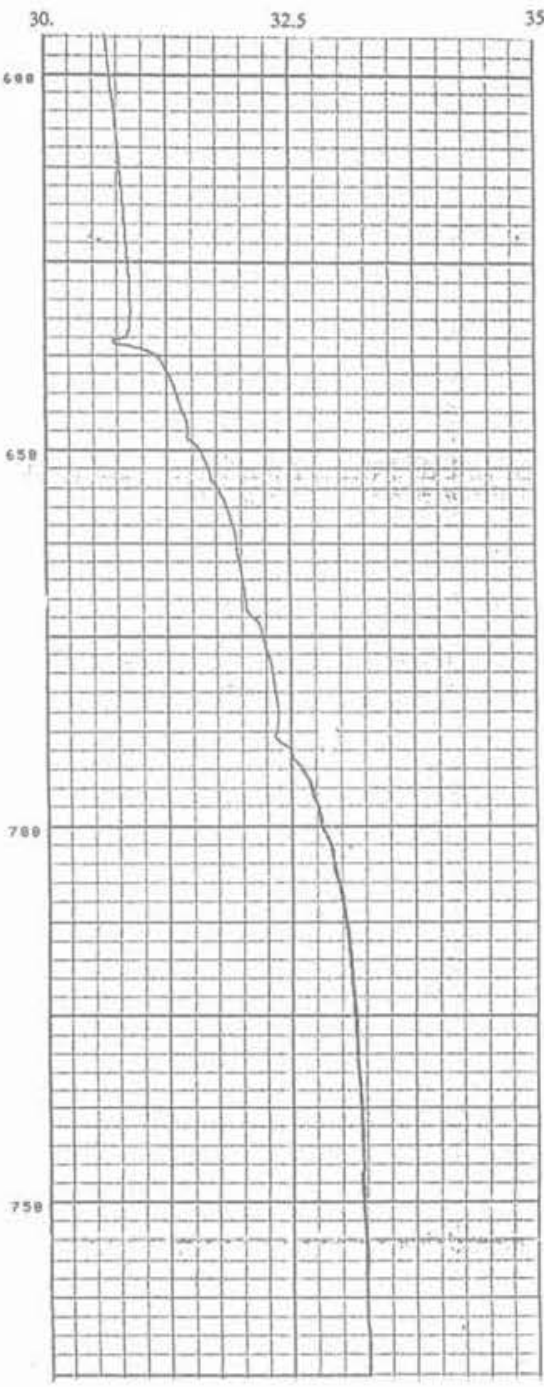

b)

Fig. 11. - Thermométrie du forage placé en condition de production; a) gradient géothermique (exprimé en degré celsius pour 10 mètres); b) température (degrés Celsius).

Fig. 11. - Thermal log of the well under production

conditions. a) Geothermal gradient (expressed in degree celsius per 10 meters) ; b) temperature (degree celsius).

variation de vitesse de rotation observée à cette cote lors de la débitmétrie a une autre cause. Celle-ci pourrait être la petite variation de vitesse de remontée du micro-moulinet intervenue à cette cote (de l'ordre de $0.5 \mathrm{~m} / \mathrm{min}$.).

Aucune venue d'eau notable n'est observée entre $700 \mathrm{~m}$ et $770 \mathrm{~m}$, cote du début de la diagraphie. Cependant l'absence de gradient thermique mesuré entre $770 \mathrm{~m}$ et $755 \mathrm{~m}$ suggère une forte venue d'eau en-dessous de. $770 \mathrm{~m}$.

Enfin on relève une forte anomalie thermique à $473 \mathrm{~m}$ à l'intérieur du tubage, signe d'une venue d'eau importante dans l'annulaire à cette profondeur.

Ces résultats sont donc en accord avec la débitmétrie réalisée lorsque le forage était en condition d'injection. Cependant vis-à-vis des débitmétries on remarque la bien meilleure précision des diagraphies thermiques pour identifier les cotes où interviennent les venues d'eau. Un travail est en cours pour essayer de déduire de ces anomalies thermiques le débit d'écoulement dans la fracture correspondante.

\section{CONFRONTATION DES RÉSULTATS}

Une confrontation des résultats obtenus avec les diverses diagraphies réalisées est présentée dans le tableau 2. Pour lanalyse des cuttings nous avons reporté toutes les cotes identifiées comme des zones de fractures ouvertes ou cimentées. Pour la géochimie des eaux, seules les cotes identifiées par BIDAUX (1987) comme lieu de venues d'eau possibles ont été indiquées. Pour les diagraphies thermiques du forage au repos, nous avons regroupé les mesures effectuées avant et après les essais de fracturation hydraulique à $441 \mathrm{~m}$. Nous avons reporté toutes les cotes pour lesquelles le gradient s'écartait de plus de $5^{\circ} \mathrm{C} / 100 \mathrm{~m}$ par rapport à la valeur moyenne. Pour les diagraphies électriques nous avons considéré le laterolog SCHLUM. BERGER et avons retenu toutes les cotes où des résistances inférieures ou égales à $100 \mathrm{ohms}$ avaient été mesurées. En ce qui concerne le Darcilog, nous avons retenu toutes les cotes où l'indice I dépasse la valeur 20 (rappelons que plus l'indice $\mathrm{I}$, adimensionnel, est élevé plus le milieu est supposé perméable). La valeur maximum de 40 a été atteinte à $672 \mathrm{~m}$. Pour les diagraphies d'atténuation des ondes $\mathrm{S}$ et d'atténuation des ondes de STONELEY nous avons retenu, comme pour les diagraphies pécédentes, un seuil arbitraire mais suffisamment élevé pour mettre en évidence les cotes où des valeurs extrêmes apparaissent.

Ainsi, bien que ces différents seuils soient arbitraires, ils permettent, en précisant les cotes où les valeurs extrêmes sont observées, d'identifier la (ou les) diagra. phie(s) susceptible(s) de mettre en évidence les zones de fracture les plus significatives du point de vue des écoulements forcés dans le massif.

D'après le tableau 2, il apparaît que si les diagraphies électriques permettent de bien déterminer au niveau du forage la géométrie des fractures dans lesquelles interviennent des écoulements (log MOSNIER), elles ne permettent absolument pas de prévoir les zones les plus hydrauliquement permissives. En effet, les cotes où la résistance électrique mesurée est très faible ne coincident pas généralement avec les cotes où de forts écoulement sont intervenus. Ainsi, la fracture à $473 \mathrm{~m}$ qui joue un rôle majeur en ce qui concerne l'écoulement dans le massif, comme nous l'avons vu avec les diverses diagraphies thermiques et la diagraphie de géochimie des eaux, n'apparaît pas sur les diagraphies électriques comme un élément de grande importance même si elle est bien identifiée. Cette remarque est également valable pour les fractures à $635 \mathrm{~m}$ et à $268 \mathrm{~m}$. 
TABLEAU II. - Confrontation des résultats obtenus dans le forage INAG III-8. Nous avons reporté toutes les cotes pour lesquelles la diagraphie correspondante est supposée indiquer une fracture. Z est un simple indicateur d'échelle de profondeur (en mètres).

TABLEAU II. - Results obtained in the granitic well INAG III-8. For each log, depths are indicated for which natural fractures are supposed to occur. On the extreme left, $Z$ is only a depth scale.

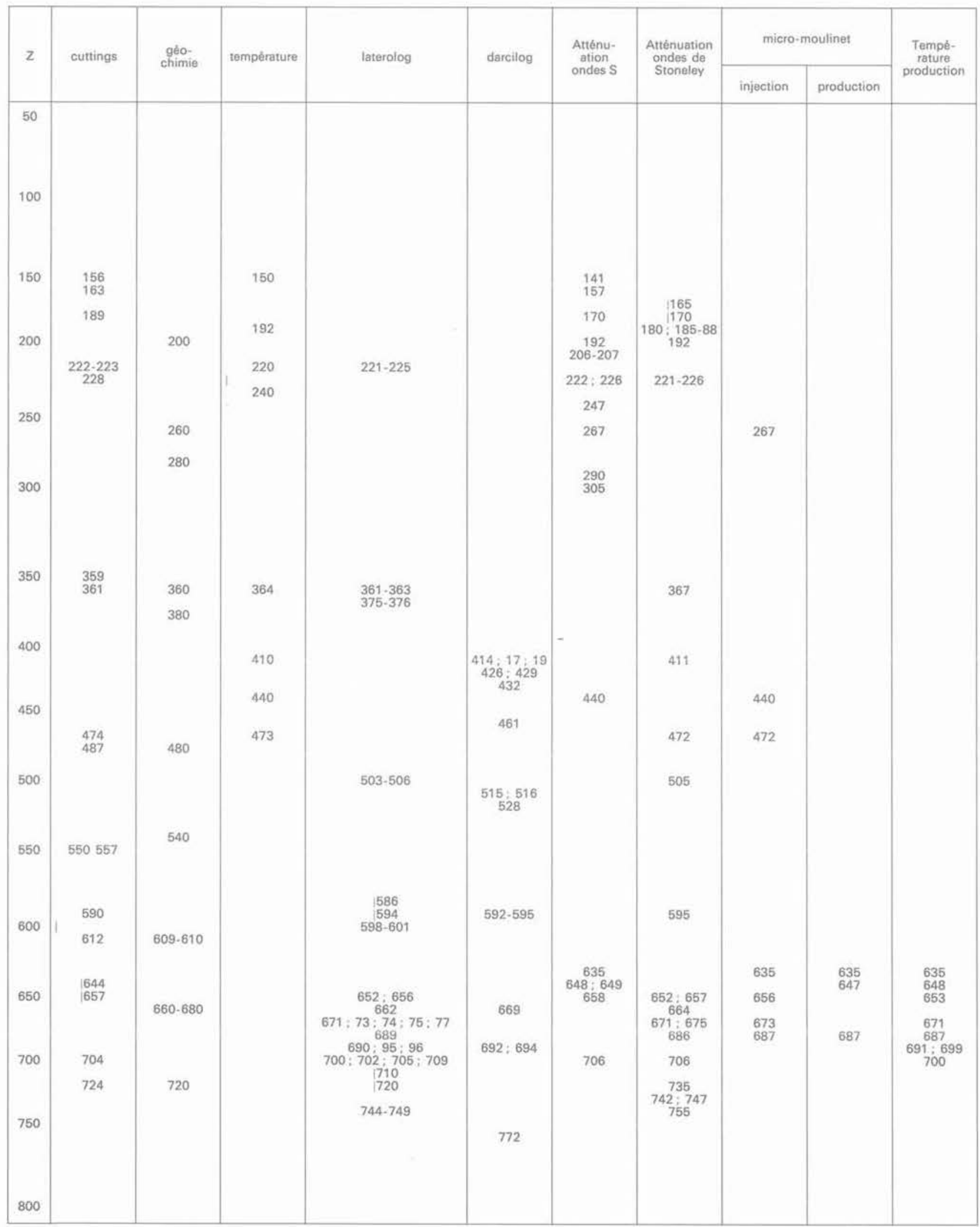


Pour la diagraphie de géochimie des eaux, du fait de la distance entre les points d'échantillonnage, on peut considérer que les cotes $267 \mathrm{~m}, 471 \mathrm{~m}$ et $673 \mathrm{~m}$ mises en évidence sur les débitmétries correspondent à des fractures où interviennent des circulations naturelles. Toutefois les cotes 360 - $380 \mathrm{~m}, 540 \mathrm{~m}, 609$ $610 \mathrm{~m}, 720 \mathrm{~m}$ identifiées par la géochimie ne correspondent à aucun écoulement notoire lors des essais d'injection ou de production. Réciproquement les cotes $635 \mathrm{~m}, 648 \mathrm{~m}, 653 \mathrm{~m}, 691 \mathrm{~m}, 699$ - $700 \mathrm{~m}$, identifiées sur la diagraphie thermique lorsque le forage était en condition de production n'apparaissent pas sur les diagraphies chimiques. Ce résultat montre ainsi que toutes les fractures perméables connectées au réseau de fractures naturelles ne sont pas nécessairement le siège d'écoulement naturel détectable. On peut également conclure que les perturbations chimiques de l'eau du forage ne sont pas uniquement causées par des venues d'eau mais résultent parfois de variations de composition chimique de la matrice rocheuse (cote $540 \mathrm{~m}$ par exemple).

Pour le Darcilog, il est certain que la valeur la plus élevée de l'indice I coincide pour l'intervalle de profondeur considéré, à la zone d'impédance hydraulique la plus faible. Toutefois on relève de très nombreuses cotes où l'indice I est élevé mais pour lesquelles aucun écoulement d'eau n'a été mis en évidence (414 à $432 \mathrm{~m}, 515 \mathrm{~m}, 516 \mathrm{~m}, 528 \mathrm{~m}, 592$ à $615 \mathrm{~m})$. Enfin la fracture à $472 \mathrm{~m}$ qui est l'une des plus significatives du point de vue hydraulique, n'a pas été identifiée avec le Darcilog.

La diagraphie d'atténuation des ondes $\mathrm{S}$ a bien mis en évidence les cotes $267 \mathrm{~m}, 441 \mathrm{~m}, 635 \mathrm{~m}$ qui correspondent aux cotes où cette atténuation atteint des valeurs extrêmes. Toutefois, avec le seuil arbitraire choisi pour faire ressortir les valeurs élevées, la fracture à $472 \mathrm{~m}$ n'a pas été identifiée. Sur les sept zones perméables détectées lorsque le forage était en injection, quatre ont été identifiées mais trois sont passées inaperçues. Par contre de nombreuses autres cotes ont été soulignées qui n'ont pas donné lieu à des écoulements détectables. Des conclusions comparables sont tirées de la diagraphie d'atténuation des ondes de STONELEY : si cette diagraphie a bien fait ressortir la fracture à $472 \mathrm{~m}$, elle n'a pas permis d'identifier les cotes $267 \mathrm{~m}, 441 \mathrm{~m}, 635 \mathrm{~m}$ et $647 \mathrm{~m}$. De même elle a atteint des valeurs élevées dans des zones où aucun écoulement n'a été décelé.

\section{CONCLUSION}

Il apparaît ainsi que si la détermination du pendage et de l'azimut des fractures recoupant un forage en milieu granitique se fait bien par diagraphie électrique, aucune des diagraphies utilisées n'aura permis de caractériser de façon satisfaisante les propriétés hydrauliques des fractures. Cependant, en couplant les diagraphies d'atténuation des ondes $S$ et celles d'atténuation des ondes de STONELEY, toutes les fractures significatives ont été identifiées mais d'autres zones sans importance pour les écoulements forcés dans le massif ont également été soulignés. Avec ces deux diagraphies, il n'a pas été possible de préciser parmi l'ensemble des fractures individualisées celles qui sont les plus conductrices du point de vue hydraulique.

L'observation la plus marquante qui ressort de ces divers résultats est le contraste entre le grand nombre de fractures mises en évidence par les diverses diagraphies et par l'étude détaillée des cuttings et le petit nombre de niveaux d'écoulement d'eau détectés, que ce soit lorsque le forage était en condition d'injection ou lorsqu'il était en condition de production. Il est même apparu lors des études menées sur le forage III9 que des niveaux identifiés comme producteurs d'eau lors de la réalisation du forage se sont révélés être sans importance lors des débitmétries.

Ainsi, pour déterminer les niveaux d'écoulement les plus significatifs dans le massif il ne suffit pas de mettre en évidence les fractures les plus conductrices au niveau du forage, il est aussi nécessaire de connaître l'interconnection de ces fractures dans le massif. Ce point paraît beaucoup plus délicat à explorer avec les diagraphies classiques et seules les débitmétries ont permis de fournir des informations à ce sujet pour les essais du Mayet de Montagne.

\section{REMERCIEMENTS}

Ce travail a été financé par le Ministère de la Recherche et de la Technologie (fonds de la Recherche - secteur génie civil), l'Agence Française pour la Maitrise de l'Energie, le Centre National de la Recherche Scientifique (INSU et PIRSEM) et la Commission de Communautés Européennes (DG XII - Géothermie). Je tiens à remercier la société Elf Aquitaine et plus particuliè. rement $\mathrm{P}$. Arditty et $\mathrm{F}$. Mathieu qui m'ont communiqué leurs résultats obtenus avec la sonde EVA.

\section{BIBLIOGRAPHIE}

BATCHELOR A.S. (1984), Hot Dry Rock Geothermal Exploitation in the United Kingdom. Modern Geology, vol. 9 , nb.1, p. 1-43, Gordon and Breach Science Publishers.

BEYDOUN W.B., CHENG C.H. and TOKSÖZ M.N. (1985), Detection of open fractures with vertical seismic profiling. Journal Geophys. Res., vol. 90, nb. B6, pp. 4557-4566.

BIDAUX P. (1987), Contribution à l'étude des circulations profondes en milieu fissuré peu perméable, identification à partir de mesures hydrochimiques le long de forages. Thèse de Doctorat de l'Université de Montpellier, spécialisé Géologie.

BIOT M.A. (1956), Theory of propagation of elastic waves in a fluid saturated porous solid. Jour Acoustic Soc. Am., vol. 28, pp. 168-191.

CHENG C.H. and M.N. TOKSÖZ. (1981), Elastic wave propagation in a fluid filled borehole and synthetic acoustic logs. Geophysics, vol. 46, pp. 1042-1053.

CONCHE B. (1986), Apport des diagraphies acoustiques dans l'identification de la fracturation en forage: modélisation, étude de cas concrets. Mémoire de diplôme d'ingénieur Géophysicien de Strasbourg. 
CORNET F.H. et JULIEN Ph. (1987), Stress determination from hydraulic tests data and focal mechanisms of induced seismicity. Soumis pour publication à Int. Jour. Rock Mech. Min. Sc.

COUTURIE J.P., BINON M. et CARMIER F. (1984), Rapport géologique sur le forage INAG III-8; Rapport "Mayet de Montagne ». F.H. Cornet, lnst. de Phys. du Globe de Paris.

DUCOMTE C. et CONRAD R. (1984), Application d'une diagraphie de perméabilité «Sealdex et Exaflo $» 9^{\circ}$ colloque international de diagraphies, Paris, Soc. Av. Int. Diagraphies.

HARDIN E.L., CHENG C.H., PAILLET F.L and MENDELSON J.D. (1987), Fracture characterization by means of attenuation and generation of tube waves in fractured crystalline rock at Mirror Lake, New Hampshire. Jour.Geophys. Res., vol. 92, nb B8, pp. $7989-8006$.

JOLIVET J. (1985), Etude thermique du sondage III-8, Rapport Mayet de Montagne 1/10/83 - 1/7/87. F.H. Cornet, Institut de Physique du Globe de Paris.

LEBRETON F., SARDA J.P., TROQUEME E. et MOR. LIER P. (1978). Logging tests in porous media to evaluate the influence of their permeability on acoustic waveforms. 19 th S.P.W.L.A. Logging Symp. ; El Paso.
MATHIEU F. and TOKSÖZ M.N. (1984), Application of full waveform acoustic logging data to the estimation of reservoir permeability. Paper BHG 15; 54 th S.E.G. meeting, Atlanta.

MATHIEU F. et ARDITY P. (1986), Rapport interne Elf-Aquitaine. Département sismique de puits, ParisLa Défense.

MOSNIER J. (1982), Détection électrique des fractures naturelles ou artificielles dans un forage. Annales Géophys., vol. 38, pp. 537-540.

PAILLET F.L. and WHITE J.E. (1982), Acoustic modes of propagation in the borehole and their relationship to rock properties. Geophysics, vol. 47, pp. $1215-1228$.

PEYREL J.Y. (1972), Pétrologie des granites rouges du N.E. du Massif Central francais. Thèse $3^{\mathrm{c}}$ cycle, Univ. Clermont-Ferrand II, $152 \mathrm{P}$.

RASALOFOSAON P. (1987), Propagation des ondes acoustiques dans les milieux poreux. Effets d'interface. Thèse de Docteur es-sciences. Univ. Paris VII.

ROSENBAUM J.H. (1974), Synthetic microseismogram: logging in porous formation. Geophys. vol. 39 , pp. $14-32$.

WHITE J.E. (1983), Underground sound, 252 pp., Elsevrier, Amsterdam. 